\title{
Improved Method for Long-Term Frequency Stability Measurement Using Vector Voltmeter
}

\author{
Ivica MILANOVIC, Zeljko BELJIC, Predrag RAKONJAC, Zoran MITROVIC
}

\begin{abstract}
This paper proposes a novel approach for the improvement of the long-term frequency stability measurement using an obsolete phase meter. The hypothesis of the paper states it is possible to fully automate measurement process and reduce measurement time using the standard method supported by bespoke PC software. This theory was examined in detail and experimentally validated using GPS standard method. The results of the experiment confirmed that measurement time can be reduced more than $50 \%$, at the expense of a measurement uncertainty increase. Still, the estimated measurement uncertainty of the proposed method is more than satisfactory for the calibration purpose of the most common oscillator types including rubidium frequency standards.
\end{abstract}

Keywords: automation; frequency; long-term stability; phase angle; vector voltmeter

\section{INTRODUCTION}

Frequency stability is the most important characteristic of an oscillator. Stability does not specify the accuracy of the frequency, rather, it shows to what extent the frequency is stable during the observed time interval. If we consider time intervals of one hour, day, month or a year, then we talk about the long-term stability [1].

Stability is defined as a statistical estimation of the frequency or time fluctuations of a signal, over a given time interval. Statistical estimations can be presented in the frequency domain or, more often, in the time domain. In order to determine frequency stability in the time domain, we have to measure a set of frequency offsets and to calculate scattering of the collected data. The measure of frequency stability in the time domain is nonoverlapping Allan, or two-sample variance [2]. It is described as:

$\sigma_{y}^{2}(\tau)=\frac{1}{2(M-1)} \sum_{i=1}^{M-1}\left(\bar{y}_{i+1}-\bar{y}_{i}\right)^{2}$

where $\sigma$ is Allan Variation, $\tau$ is averaging time, $M$ is the number of fractional frequency values $(\Delta f / f)$, and $y_{i}$ is the $i^{\text {th }}$ of $M$ fractional frequency data, averaged over the averaging period $\tau[2,3]$.

While standard deviation subtracts the mean from each measurement before squaring their sums, the Allan deviation subtracts the previous data point from the current one. This differencing of successive data points removes the time dependent noise contributed by the frequency offset.

The stability is being improved as the averaging time $(\tau)$ gets longer, because, in some cases, noise and interferences can be removed by averaging [1]. However, on some level, further averaging no longer improves the results [4].

In order to improve the confidence of a stability estimate, the calculation described with Eq. (1) can be carried out by utilizing all possible combinations of data sets. This is the so-called overlapping method:

$\sigma_{y}^{2}(\tau)=\frac{1}{2 m^{2}(M-2 m+1)} \sum_{j=1}^{M-2 m+1} \sum_{i=j}^{j+m-1}\left(\bar{y}_{i+m}-\bar{y}_{i}\right)^{2}$, where $\sigma$ is Overlapped Allan Variation and $\mathrm{m}$ is averaging factor $\left(\tau=m \cdot \tau_{0}\right)$. The Overlapping Allan Variation presents the standard measure for frequency stability in the time domain [2].

For example, to calculate stability for $\tau=10$ minutes with $\tau_{0}=1 \mathrm{~min}$, the non-overlapping method considers successive data sets: from $1^{\text {st }}$ to $10^{\text {th }}$, then $11^{\text {th }}$ to $20^{\text {th }}$, etc. On the other hand, the overlapping method uses all possible combinations of 10 -samples data sets: from $1^{\text {st }}$ to $10^{\text {th }}, 2^{\text {nd }}$ to $11^{\text {th }}, 3^{\text {rd }}$ to $12^{\text {th }}$, etc. In that way a better statistical uncertainty and signal/noise ratio are provided. The aim of the measurement is to determine y data set. There are a few standard methods for long-term stability measurement:

- Direct method with time interval or frequency counters [2]

- Heterodyne method [2]

- Dual mixer time difference method [2]

- Method based upon GPS time transfer standards [5]

- Method with modern time interval analysers [6].

Direct method is suitable to determine 24-hour stability. Still, because of its inadequate measurement uncertainty, it is unusable for ultra-stable quartz, rubidium or caesium beam disciplined oscillators. On the other hand, the GPS receiver provides excellent capabilities. However, only half-hour samples are available for further data analysis, and tracking time lasts at least one week, or even longer.

Regarding measurement uncertainty, heterodyne and dual mixer methods are between direct and GPS controlled methods, but they demand constant use of various measurement equipment, such as mixers, amplifiers, LO generators [2], etc. Those facts make them hard to use, especially for measurement time of several days. For measurement of oscillator's long-term stability, our laboratory uses the standard method - based on the GPS transfer standards for frequency and time (Allen Osborne Associates TTR-6). By employing this method, it is possible to determine the characteristics of the most stable commercial oscillators, such as caesium standard. As mentioned above, the output data are half-hour samples of the measured phase difference. To obtain the satisfactory amount of data for statistical analysis of stability, minimal measuring time is 7 days, and in some 
cases 14 days, regardless of oscillator nominal stability. However, the greatest number of calibration requests refers to quartz and rubidium standards, which do not require such rigorous measurement uncertainty.

On the other hand, another standard method based on a vector voltmeter is practically useless, since it uses an obsolete chart recorder for data acquisition, and data analysis is performed manually. Except for any discomfort during the regular operation, the probability of human error is very high. The main motivation for the revision of this method is to reduce the overall measurement time as much as possible in accordance with the required measurement uncertainty, thus to automate the entire measurement system in order to eliminate the influence of human errors.

In order to improve the measurement procedure (i.e. to reduce measurement time and to eliminate human factor as much as possible), a novel method based on a vector voltmeter has been developed. It provides a suitable measurement uncertainty for quartz or rubidium oscillators' long-term stability measurements. Within three days period, the sufficient amount of data can be collected for a quality analysis. The old-fashioned equipment is used for development of a very efficient, flexible and user-friendly method. Measurement process is controlled by a PC, consequently allowing easy data analysis and reports generation.

\section{PHASE DIFFERENCE MEASUREMENT METHOD}

The goal of frequency stability analysis is to characterize the phase and frequency fluctuations of a frequency source [2]. This kind of measurement can be realized if we compare its phase with the phase of the reference signal [7]. Determination of frequency stability, based on two sine-wave signals phase difference measurements, can be presented with block diagram in Fig. 1:

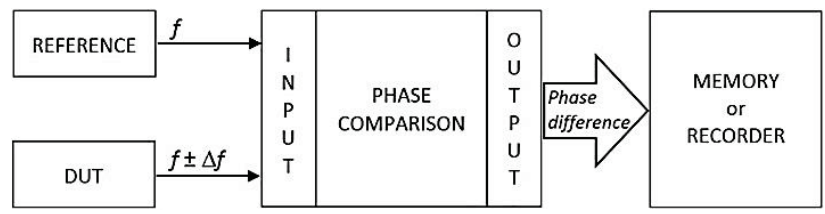

Figure 1 Frequency stability measurement - phase difference method

In order to measure stability of the Device under test (DUT) signal, we have to perform a set of phase difference measurements, equally distributed in time, and to collect measurement results for further analysis. The aim is to determine the frequency offset, and to present it in the time domain.

The phase angle between two signals changes by 360 degrees every second for each cycle $(\mathrm{Hz})$ of their difference in frequency [8]. Stated in another form:

$$
\frac{360^{\circ}}{1^{\circ}}=\frac{\frac{\Delta \varphi}{\Delta t}}{\Delta f},
$$

where $\Delta \varphi$ is the measured phase difference, $\Delta t$ is the time interval during $\Delta \varphi$ are measured, and $\Delta f$ is the frequency difference between two signals. Eq. (3) can be presented as:

$$
\Delta f=\frac{\Delta \varphi}{360^{\circ} \cdot \Delta t} .
$$

Further:

$$
\frac{\Delta f}{f}=\frac{\Delta \varphi}{360^{\circ} \cdot \Delta t \cdot t}
$$

Symbol $f$ presents the nominal frequency of signals (in $\mathrm{Hz}$ ), and $\Delta f / f$ is the desired difference (ratio) of frequency offsets, occurring in $\Delta t$ period (in seconds). $\Delta \varphi$ data are expressed in degrees.

If we know the nominal frequency and perform phase difference measurements in equal $\Delta t$ intervals, we can calculate the frequency offset using the formula (5).

\section{THE BASIC IDEA AND IMPROVEMENT OF THE STANDARD METHOD}

One of the instruments for instant phase difference measurement is the vector voltmeter HP 8405A. It compares the reference signal on input $\mathrm{A}$, and the DUT signal on input $\mathrm{B}$. The automatic phase control (APC) uses a phase-locked loop to tune and a phase-lock meter to measure the channel A signal. The APC adjusts frequency of voltage-tuned local oscillator, which triggers sampling-type mixers. The RF signals are reconstructed from the samples at intermediate frequency of $20 \mathrm{kHz}$, where voltage and phase are measured [9].

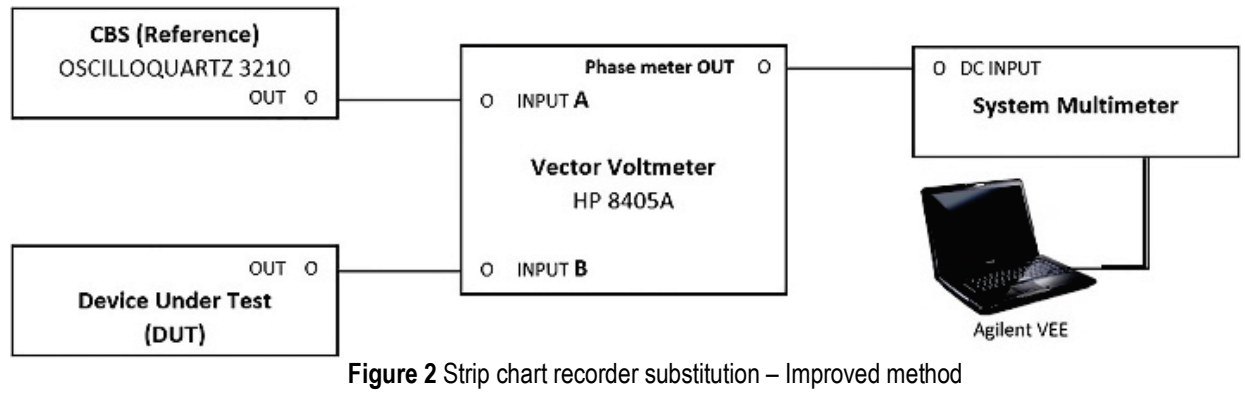

The old method implies the use of a strip chart, which continuously collects phase difference data, measured by the vector voltmeter. In further analysis, the results have to be manually read out from the chart. Due to a large number of data, it can be very difficult and time consuming process. Besides, errors caused by human mistake may be common. 
In order to simplify the measurement process, the idea was to replace the existing strip chart recorder with a system multimeter and a computer, and make measurement and data analysis as independent from human influence as possible. Fig. 2 depicts the block diagram of the proposed improved method.

The measurement process is fully automated using PC. Unfortunately, the vector voltmeter has no capability to communicate with the PC (there are no interfaces like HPIB, USB, RS232, or similar). However, phase difference can be measured on its 'Phase meter' rear output: the DC voltage on this output corresponds to an instant phase difference [10], as it is presented in Fig. 3.

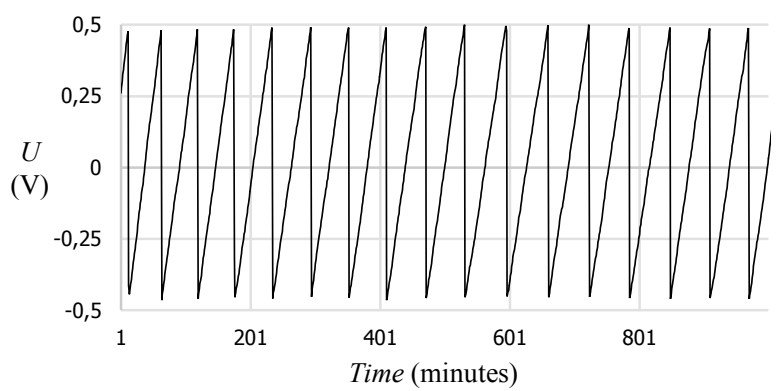

Figure $3 \mathrm{DC}$ level on vector voltmeter output, due to phase changes, example

For DC voltage measurement, a digital multimeter or a system multimeter is used.

The vector voltmeter HP 8405A can achieve the highest accuracy if it compares signals with equal levels [11]. Therefore, before measurement, we have to attenuate DUT and reference signals to achieve equal or approximately equal levels on vector voltmeter inputs.

For very stable frequencies (oven controlled quartz oscillators, rubidium or caesium beam standards) the phase difference will not exceed 12 degrees from the reference. If so, the HP $8405 \mathrm{~A}$ can be switched to its $-6^{\circ}$ to $+6^{\circ}$ range. That option should be used whenever possible, because it results in significantly better measurement uncertainty.

\section{AUTOMATION SOFTWARE OVERVIEW}

For the purposes of measurement process automation a bespoke software application has been developed using the Agilent VEE Pro (Visual Engineering Environment) software. A connection between the measuring equipment and a PC is realized using the GPIB/USB interface. The software is designed for collecting measurement data, their processing and a report generation. To avoid undesirable delays or data overflow during the communication, the program is divided into three separate parts (stages):

- configuration of the measurement,

- data acquisition, and

- data processing accompanied by a report generation. Fig. 4.

The functional model of the program is illustrated in

In the configuration part, the user defines the nominal frequency and the tracking time. The software then calculates an optimal sample rate in order to avoid large amount of data, which could give rise to problems in subsequent calculations and processing.

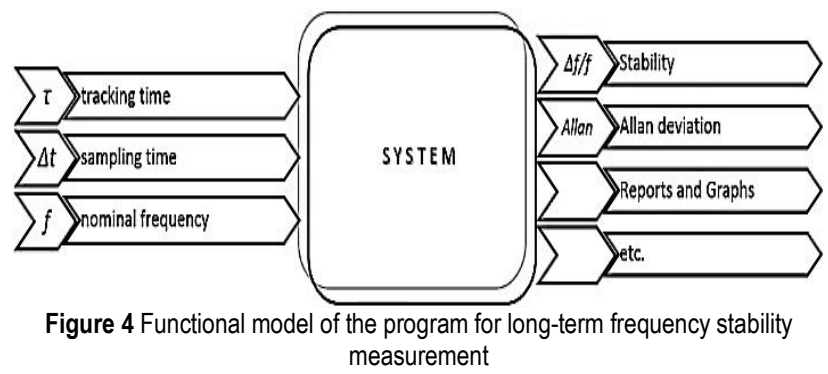

To the end of the measurement, the process is independent of the human interference, and therefore a possibility for human-caused error is eliminated.

The acquisition part of the program only collects measured $\Delta \varphi$ data, without any statistical or mathematical analysis. After the acquisition is finished, the raw phase difference data are stored in PC's storage, and the data processing begins.

The data processing includes:

- Volt to degree data transformation,

- Data correction (Volt to degree conversion and outliers removal) [4],

- Calculation of $\Delta f / f$,

- Determining of long-term stability, and

- Calculation of Overlapped Allan Variation.

The program calculates the mean value of 24-hour $\Delta f / f$ data, and their differences. The long-term stability presents the worst case of calculated frequency differences.

At the very end, a report is generated as a bit map (.bmp) figure, however, the raw data and the intermediate results are stored in text files and can be used for further analysis.

The Front Panel of the software and the report views are presented in Figs. 5, 6 and 7-respectively.

In Fig. 5 a user interface, or first panel view, is presented. The user defines measurement configuration: nominal frequency, tracking (measurement) time, and basic DUT information, needed for making the final report using this interface. After data entering, the measurement begins, and the final result is the Calibration report. An example of the report is presented in Fig. 6.

Fig. 6 shows a Calibration Report example. Besides the DUT info, the measured stability and the phase difference chart are presented, too. The values of Allan deviation, calculated using Eq. (2), and the Allan deviation chart, as a part of the Calibration Report, are presented in Fig. 7.

Allan deviation diagram is presented as a log sigmatau diagram. The horizontal axis shows time in seconds, while the vertical axis presents the value of overlapping Allan's deviation, as a multiple of $10^{-9}$.

The program is flexible and allows adaptation to various requirements. However, it was empirically concluded that the optimal monitoring period is 3 days, with the sampling time of $1 \mathrm{~min}$.

Limited resolution of the measuring system and size of the internal noise, limit its overall capabilities. That 
means that the minimal sampling time is 1 second. As the aim of this method is to determine signal stability for a day, the experimental measurements have shown that oneminute measurements over the three days period give acceptable results.
Longer time measurements and more frequent sampling certainly decrease the total measurement uncertainty. However, the experimental measurements have shown that an increase in total measurement time does not reduce measurement uncertainty significantly, while the results stay practically the same.

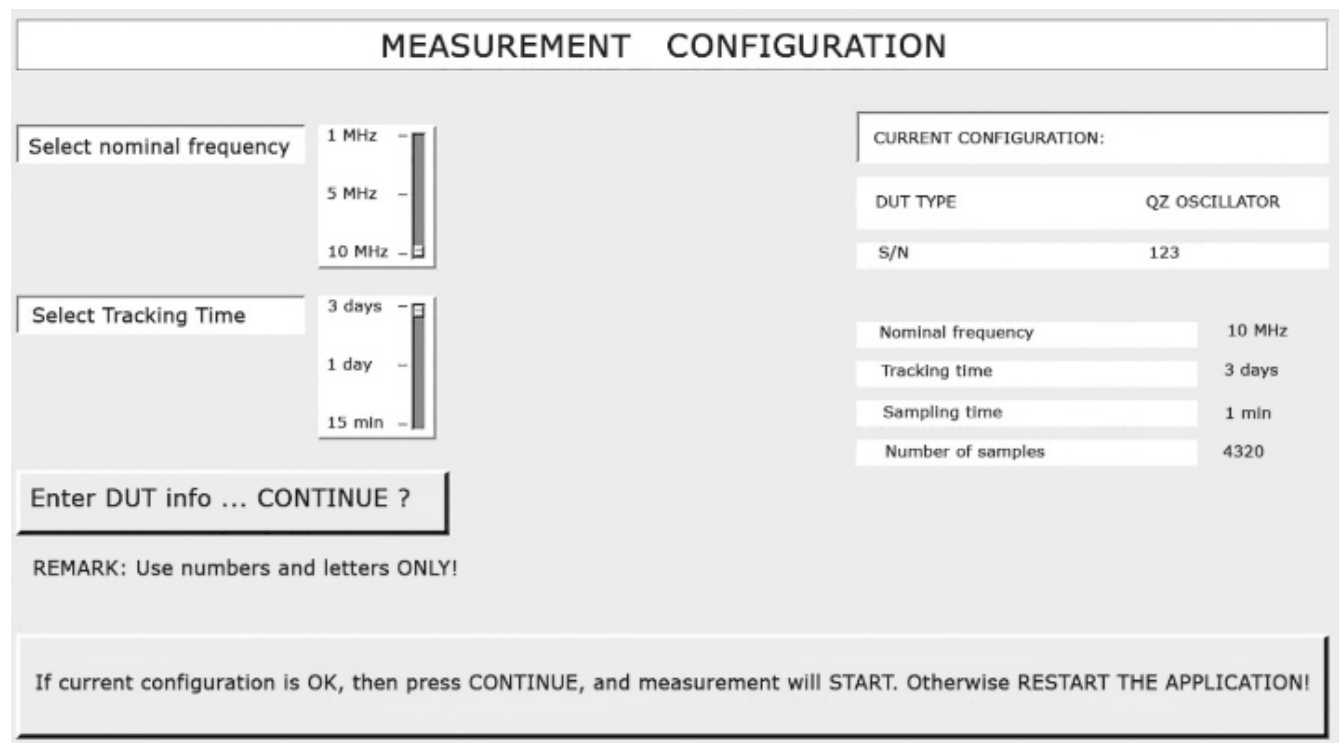

Figure 5 Application - Front Panel view, example

\section{CALIBRATION REPORT}

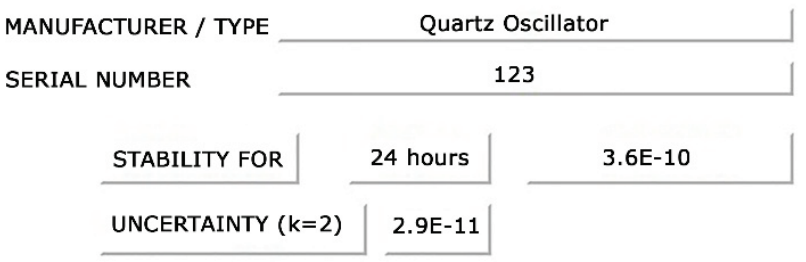

PHASE DIFFERENCE CHART

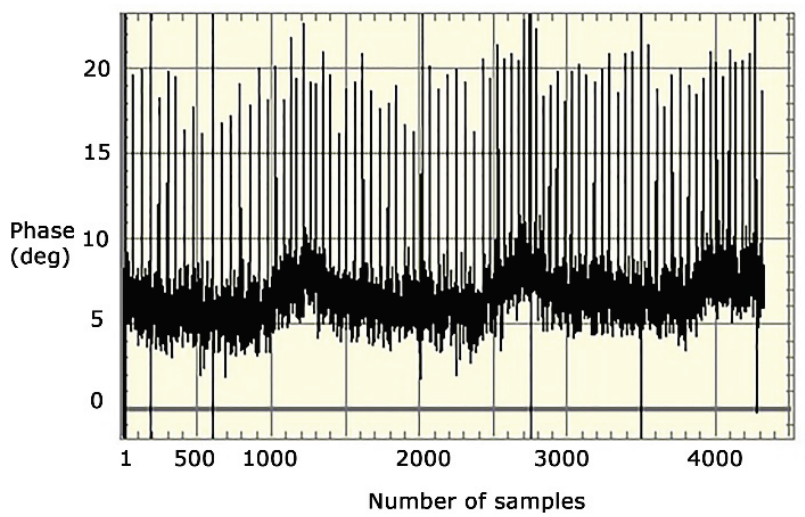

Figure 6 Application-calibration report view, example

In the case of increase in the time and number of measuring, second problem can be data processing of the large number of the obtained data, due to personal computer capabilities (processor speed, RAM, etc.).

The experiments in our laboratory have shown that, for example, 10 days measurements with 1 hour sampling time, give approximately the same results of signal stability for 1 day.

The analysis of stability and Alan's deviations were carried out using predefined functions embedded into the
VEE package and using MATLAB scripts created within the VEE. The software is validated by comparing the outputs of mathematical functions of the program against the results obtained using commercial software for the analysis of frequency stability - Stable32 [12].

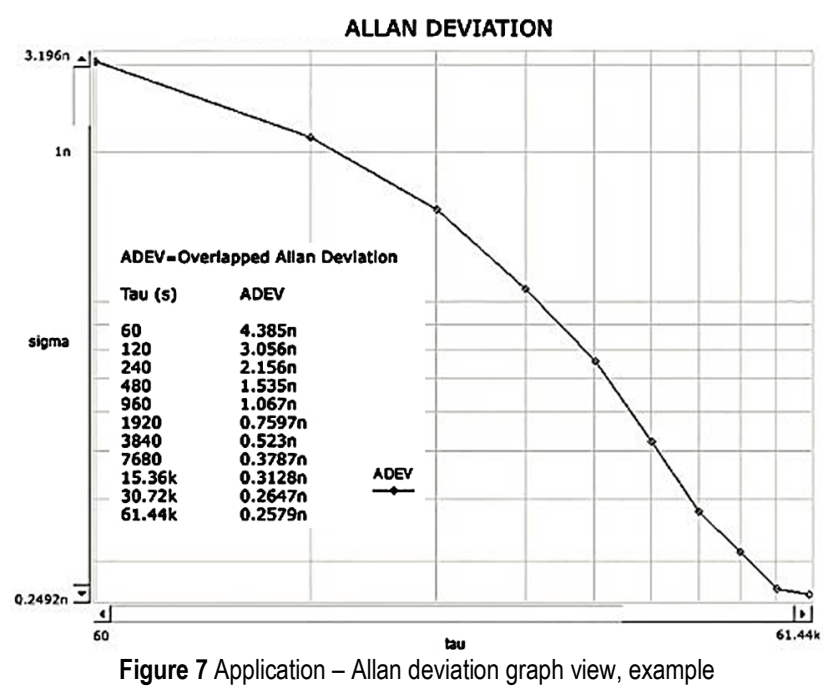

Raw data, their conversion, all the calculations, as well as the final results, are stored in separated text files placed in computer's storage. This allows complete access to data, making it possible to execute process control and thus leaves the possibility for further analysis and research.

This method is characterized by simplicity of use, continuous control of the measurement process and complete automation of long-term frequency stability measurement. Incorporating a PC into measurement process provides virtually unlimited possibility for storing data and measurement results. 


\section{MEASUREMENT UNCERTAINTY}

Assuming Eq. (5), a mathematical model for signal stability has been designed, as it is presented in Eq. (6):

$y_{i}=\frac{\delta \varphi+\delta \varphi_{\mathrm{trac}}+\frac{\left(v+\frac{v \cdot \delta T}{T}+\delta v_{r}+\delta v_{V}+\delta v_{\theta}\right) \cdot\left(\delta \varphi+\delta \varphi_{\mathrm{trac}}+\varphi_{R}\right)}{v_{R}+\delta v_{R}}}{c \cdot(T+\Delta T+\delta T) \cdot\left(f_{N}+\Delta f_{N}+\delta f_{N}\right)}$.

To determine a measurement uncertainty budget, a program in Mathematica has been made. This code allows experimentation by changing certain parameters of the measurement uncertainty budget. Here will be shown three examples, where we used different phase measurement ranges, defined on the vector voltmeter. Measurement uncertainty budgets are created in accordance to the generally accepted European documents [13] and [14]. Also, this experiment is used for the validation of the proposed method and performed calculations.

Let us assume that the sampling time $\Delta t$ from Eq. (5) is 1 minute: $\Delta t=T=60 \mathrm{~s}$. In Eq. (6) $y_{i}$ presents relative frequency difference between the DUT and the reference signal, in the i-th minute of a measurement. $\varphi_{R}$ is a range of a phase measurement, selected on the vector voltmeter, and can be set to $\pm 6, \pm 18$ or \pm 180 degrees [10]. In the whole phase measurement range, the DC voltage on the vector voltmeter output spans from $-0.5 \mathrm{~V}$ to $+0.5 \mathrm{~V}$ [10] That DC voltage range is presented by $v_{R}$ in the actual model. The correction of $v_{R}$ is experimentally determined by observing data at the ends of the voltage range. This correction is given by $\delta v_{R}$. The expected value of $\delta v_{R}$ is $4.5 \mathrm{mV}$, and the standard uncertainty is $3.3 \mathrm{mV}$. The normal distribution is assumed, and the measurement uncertainty is type A.

In the model (6), $v$ presents the DC voltage, measured with a digital multimeter, in the i-th minute of a measurement. Because of not knowing the exact moment when the measurement starts, we have to include the correction of level $v$, and it is given as $(v \cdot \delta T) / T$. When the system gives instruction for $v$ level measurement, it starts after $\delta T$, until the instruction is performed. We can assume that the total unforeseen delays are in the interval from 0 to $2 \mathrm{~ms}$ [15]. The mathematical expectation of $\delta T$ is the mean instruction execution time. The range of possible delays can be determined indirectly. The uniform distribution, and the type $\mathrm{B}$ of the measurement uncertainty is assumed.

On the other hand, the error caused by inaccuracy of determination of $T$ is presented with $\Delta T$. It is the result of a system clock inaccuracy, which is certainly better than $10^{-6}[15]$. So, it can be assumed that $\Delta T$ is less than $1 \mathrm{~ms}$. The mathematical expectation of $\Delta T$ is 0 , and the distribution is uniform.

The vector voltmeter has the specified phase measurement accuracy of \pm 1.5 degrees [10]. In the formula (6), $\delta \varphi$ presents the correction of the measured phase difference. The mathematical expectation of $\delta \varphi$ is 0 , and the standard measurement uncertainty is equal to
$1.5 / \sqrt{3}$ degrees, type $\mathrm{B}$, with assumed uniform distribution.

$\delta \varphi_{\text {trac }}$ presents the correction of measured phase difference, due to the accuracy of phase to DC level conversion, on the vector voltmeter output, often called the tracking accuracy. That accuracy is defined as $\pm 1.5 \%$ from the phase measurement range [10]. The mathematical expectation of $\delta \varphi_{\text {trac }}$ is 0 , and the standard measurement uncertainty is $\left(0.015 \cdot \varphi_{R}\right) / \sqrt{3}$ degrees, with the uniform distribution, type $\mathrm{B}$.

The DC voltage, proportional to the instant phase difference, is measured with the digital multimeter. Correction of level $v$ due to the multimeter accuracy is $\delta v_{v}$, and it is specified as accuracy/ $\sqrt{3}$ [16], with assumed uniform distribution. Besides the accuracy, level $v$ depends on the multimeter's resolution, too. That correction is named as $\delta v_{r}$. The mathematical expectation of $\delta v_{r}$ is 0 , and the standard uncertainty is type $\mathrm{B}$, equal to resolution $/(2 \sqrt{3})$, also with assumed uniform distribution.

The influence of ambient temperature changes is presented with $\delta v_{\theta}$. For a system multimeter it is defined as a percentage of reading plus number of digits by every ${ }^{\circ} \mathrm{C}$ of temperature change [17]. Assuming the triangular distribution [18], a standard uncertainty can be calculated. The environmental conditions in the metrology laboratory are strictly controlled, and temperature is within $(23 \pm 2)^{\circ} \mathrm{C}$, so it can be neglected. The mathematical expectation and the measurement uncertainty for $\delta v_{\theta}$ are equal to 0 .

In the model (6) $f_{N}$ presents the nominal frequency of the compared signals. Correction of the reference oscillator frequency due to its accuracy $\left(\Delta f_{N}\right)$, and correction due to its instability $\left(\delta f_{N}\right)$, are determined in the last certificate of calibration.

In Eq. (6) c represents a constant, that is 360.

As an example, we can observe the case when two $10 \mathrm{MHz}$ signals are compared. The phase difference measurement is performed on $\varphi_{R}= \pm 6$ degrees range every minute, and in the $i^{\text {th }}$ minute $v$ is $0.25 \mathrm{~V}$. Using the mathematical model (6) and described uncertainty contributions, the result and total measurement uncertainty is presented in the budget Tab. 1 .

The results show that the voltmeter accuracy has the biggest influence on the total measurement uncertainty, nearly $95 \%$. Contribution of the tracking accuracy participates with only $5 \%$, while the other factors are negligible. Under the same conditions, when we use \pm 18 degrees range of a phase measurement, the final result is $42 \times 10^{-12}$, with the expanded measurement uncertainty of 
$8.2 \times 10^{-12}$. The dominance of the vector voltmeters accuracy is still the biggest, $85 \%$, while the tracking accuracy accounts for $15 \%$ of the total measurement uncertainty.

By analysing the measurement uncertainty in the case when we use range of \pm 180 degrees, we have determined that the influence of the tracking accuracy is the dominant one, with $64 \%$, while the vector voltmeters' accuracy participates with $36 \%$. The other parameters still have a negligible influence. In this particular case, the result will be $420 \times 10^{-12}$, with the expanded measurement uncertainty of $16 \times 10^{-12}$.

Table 1 Measuring Uncertainty Budget for $10 \mathrm{MHz}$ signal, 1 minute samples, \pm 6 degrees phase measurement range

\begin{tabular}{|c|c|c|c|c|c|c|c|}
\hline Value & Symbol & Estimation & $\begin{array}{l}\text { Standard } \\
\text { uncertainty }\end{array}$ & Type & $\begin{array}{l}\text { Probability } \\
\text { Distribution }\end{array}$ & $\begin{array}{l}\text { Sensitivity } \\
\text { Coefficient }\end{array}$ & Contribution \\
\hline $\begin{array}{l}\text { Correction due to accuracy of vector } \\
\text { voltmeter }\end{array}$ & $\delta \varphi$ & 0 & $866 \times 10^{-3} \circ$ & B & Uniform & $4.63 \times 10^{-12}$ & $4.01 \times 10^{-12}$ \\
\hline $\begin{array}{l}\text { Correction due to tracking accuracy of } \\
\text { vector voltmeter }\end{array}$ & $\delta \varphi_{\text {trac }}$ & 0 & $52 \times 10^{-3} \circ$ & B & Uniform & $4.63 \times 10^{-12}$ & $2.41 \times 10^{-13}$ \\
\hline $\begin{array}{l}\text { Correction of the voltage range on the } \\
\text { vector voltmeters output }\end{array}$ & $\delta v_{R}$ & $-4.5 \times 10^{-3} \mathrm{~V}$ & $3.3 \times 10^{-3} \mathrm{~V}$ & A & Normal & $-6.42 \times 10^{-16}$ & $2.12 \times 10^{-18}$ \\
\hline $\begin{array}{l}\text { Correction of measuring time, due to } \\
\text { its accuracy }\end{array}$ & $\Delta T$ & 0 & 0 & B & Uniform & $-7.08 \times 10^{-14}$ & 0 \\
\hline $\begin{array}{l}\text { Correction of measuring time, due to } \\
\text { delays }\end{array}$ & $\delta T$ & $1 \times 10^{-3} \mathrm{~s}$ & $577 \times 10^{-6} \mathrm{~s}$ & B & Uniform & $-7.08 \times 10^{-14}$ & $4.09 \times 10^{-17}$ \\
\hline $\begin{array}{l}\text { Correction of the reference oscillator } \\
\text { frequency, due to its accuracy }\end{array}$ & $\Delta f_{N}$ & 0 & 0 & A & Normal & $-4.25 \times 10^{-19}$ & 0 \\
\hline $\begin{array}{l}\text { Correction of the reference oscillator } \\
\text { frequency, due to its instability }\end{array}$ & $\delta f_{N}$ & 0 & $727 \times 10^{-9} \mathrm{~Hz}$ & B & Uniform & $-4.25 \times 10^{-19}$ & $3.09 \times 10^{-25}$ \\
\hline $\begin{array}{l}\text { DC voltage on the vector voltmeter } \\
\text { output }\end{array}$ & $v$ & $0.25 \mathrm{~V}$ & 0 & A & Normal & $6.36 \times 10^{-11}$ & 0 \\
\hline $\begin{array}{l}\text { Correction of DC voltage, due to } \\
\text { multimeter accuracy }\end{array}$ & $\delta v_{v}$ & 0 & $5.05 \times 10^{-6} \mathrm{~V}$ & B & Uniform & $6.36 \times 10^{-11}$ & $3.21 \times 10^{-16}$ \\
\hline $\begin{array}{l}\text { Correction of DC voltage, due to } \\
\text { multimeter resolution }\end{array}$ & $\delta v_{r}$ & 0 & $28.9 \times 10^{-9} \mathrm{~V}$ & B & Uniform & $6.36 \times 10^{-11}$ & $1.84 \times 10^{-18}$ \\
\hline \begin{tabular}{|l} 
Correction of DC voltage, due to \\
multimeter accuracy
\end{tabular} & $\delta v_{\theta}$ & 0 & 0 & B & Uniform & $6.36 \times 10^{-11}$ & 0 \\
\hline Relative frequency difference (result) & $y$ & \multicolumn{6}{|c|}{$14.015 \cdot 10^{-12}$} \\
\hline \multirow{2}{*}{\multicolumn{7}{|c|}{\begin{tabular}{|l|} 
Combined Standard Uncertainty \\
Expanded Uncertainty $(k=2$, confidence $95 \%)$ \\
\end{tabular}}} & $4.02 \times 10^{-12}$ \\
\hline & & & & & & & $8.03 \times 10^{-12}$ \\
\hline \multicolumn{7}{|c|}{\begin{tabular}{|l|l|} 
Final RESULT & \\
\end{tabular}} & \\
\hline
\end{tabular}

All factors, which are recognized as that they can have influence on a total measurement uncertainty, are included in this model, although, for some of them, by experience, it is known that their impacts are negligible. However, they were considered for the completeness of the results. That kind of model is suitable for "what-if" analysis, when it is possible to change all the variables, even the ones that were considered as constants.

\section{EXPERIMENTAL RESULTS AND VALIDATION}

Stability measurements are performed over a few most commonly used oscillators, quartz, and rubidium disciplined [19]. In order to evaluate the proposed method based on a vector voltmeter, the stability was measured using the GPS time transfer standard, Allen Osborne TTR-6, too. This method can be assumed as the 'referent' method [20].

The experimental results of double oven quartz oscillator stability ( $5 \mathrm{MHz}$ output), are presented in Fig. 8. The measurement lasted for three days, with the sample period of 1 minute, which means that 4320 data samples were collected. The measurement range of the HP 8405A was set in coarse scale, from $-180^{\circ}$ to $+180^{\circ}$. Caesium frequency standard Oscilloquartz 3210 was used as a reference.

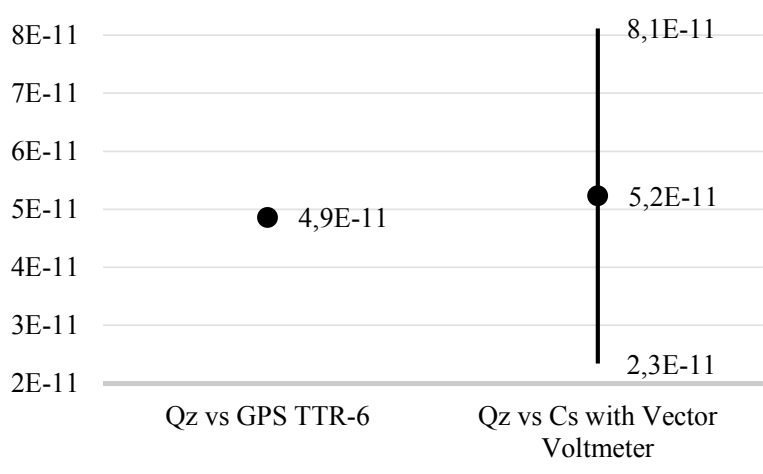

Figure 8 Experimental results - 24-h stability of double oven quartz oscillator

The measurement uncertainty of the GPS method cannot be seen in Fig. 8, because the limits are very close to the measured value. The results indicate that the method with a vector voltmeter can be used for stability measurements of ultra-stable quartz oscillators.

Further on, the experiments were carried out using a rubidium frequency standard, and using a frequency counter with rubidium time base (10 MHz outputs). Results are presented in Figs. 9 and 10.

For the purposes of this experiment, the fine range $\left( \pm 6^{\circ}\right)$ has been used, which resulted in much better measurement uncertainty. The manufacturer defines the 
stability better than $5 \times 10^{-12} /$ day. Regardless the obvious difference in stability results, the conclusion is that the vector voltmeter method can be used for rubidiumdisciplined oscillator stability measurements, as well.

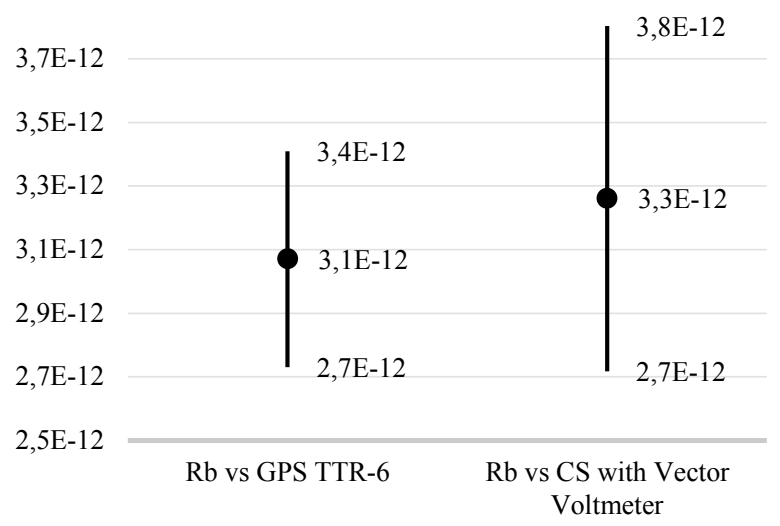

Figure 9 Experimental results -24 -h stability of rubidium frequency standard

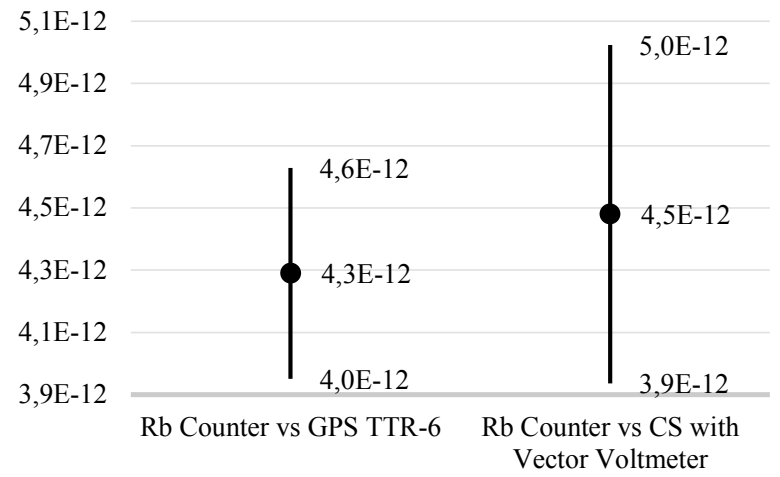

Figure 10 Experimental results - 24-h stability of frequency counter with rubidium time base

When less stable rubidium oscillator was measured (time base of the digital frequency counter) the results were, practically, identical. These experiments very clearly proved the method based on a vector voltmeter. For all three examples, the Alan deviation calculation was tested using the software for frequency stability analysis, Stable32, and there were no differences [20].

Excellent matching is also achieved for 15 minutes measuring, with $1 \mathrm{~s}$ sample time. Results are compared with the Alan deviation measured by the HP 5390A frequency stability measuring system [4]. However, this kind of measurement cannot be used for short-term stability measurements, as a consequence of the long sample period. The attempt to sample below $1 \mathrm{~s}$ time interval was not successful. The first reason was the limited speed of data transfer, and the second was noisy measurement system as a result of too high noise floor [4].

\section{CONCLUSION}

A novel approach for the improvement of the longterm frequency stability measurement using the method based on a phase meter is presented. Measurement uncertainty of the proposed method is sufficient for longterm frequency stability measurements and calibration of almost all commercial oscillators, counters, signal sources, etc. based on quartz oscillators, or even rubidium disciplined oscillators. The overall measurement interval is reduced more than $50 \%$ with acceptable measurement uncertainty, and depending on it this interval can be extended. The process of calibration is completely automated, from data acquisition to the generation of calibration reports, thus eliminating the human factor. The advantage of this method is also the large amount of useful data stored in the PC's storage, which is substantial nowadays. The entire process is completely open, making it possible to access the raw data before, as well as after processing. This raises the possibility of further analysis, such as, for example, the impact of ambient temperature changes, and much more.

The proposed method cannot replace modern compact systems for frequency stability measurements, but it can certainly be used for less demanding measurements. The modern measurement equipment are high resolution and low noise systems. Data processing is based on real-time processors. This allows undisturbed data sampling in 1 microsecond intervals. Besides long-term, this systems can precisely measure short-term stability up to 1 millisecond intervals. Measurement system described in this paper can be used for long-term stability measurement of almost all commercial oscillators which are calibrated in our laboratory. Nevertheless, experiments and measurement uncertainty calculations have shown that for calibration of high stability rubidium and caesium standards, some other methods have to be used.

\section{REFERENCES}

[1] Lombardi, M. A. (2002). Fundamentals of Time and Frequency. The Mechatronics Handbook, Chapter: 17, Publisher: CRC Press. 6, 7.

[2] Riley, W. J. (2008). Handbook of Frequency Stability Analysis, Hamilton Technical Services. NIST Special Publication 1065, July 2008, 2, 6, 14-15, 83, 95-96.

[3] Riley, W. J. (2010). The Averaging of Phase and Frequency Data, Hamilton Technical Services, November 2010 (1).

[4] Milanovic, I., Renovica, S., Zupunski, I., Banovic, M., \& Rakonjac, P. (2012). How to Measure Oscillator's ShortTerm Stability Using Frequency Counter. Electronics, 16(1), Faculty of Electrical Engineering, University of Banja Luka, June 2012, 105, 107-108. https://doi.org/10.7251/ELS1216104M

[5] Allen Osborne Associates, Inc., TTR-6/TTR-6A GPS Time Transfer Receivers Operational Manual.

[6] Symmetricom, Inc., 5110A Operations and Maintenance Manual, DOC05110A, USA

[7] Kartaschoff, P. (1978). Frequency and Time, Academic Press, London, UK, 159-164.

[8] Hewlett Packard Application Note 52, Section III, Methods for Precise Frequency Standard Intercomparison, 3-8.

[9] Technical Information from the HP Laboratories. (1966). Hewlett Packard Journal, 17(9), 3.

[10] Hewlett Packard, Vector Voltmeter 8405A Operating and Service Manual (I-2).

[11] Hamza, G. G. (2014). Investigation of the Optimum Trigger Level in Time Interval Measurement. MAPAN Journal, 29(4), 1. https://doi.org/10.1007/s12647-014-0102-4

[12] Hamilton Technical Services, Stable32, Frequency Stability Analysis, User Manual, Version 1.54, printed in 2008, 57-59. 
[13] EA - European co-operation for Accreditation, EA-4/02M, Publication Reference, Expression of the Uncertainty of Measurement in Calibration, December 1999.

[14] BIPM, IEC, ISO, Guide to the Expression of Uncertainty in Measurement, ISBN 92-67-10188-9, 1995, first edition 1993, corrected and reprinted 1995, International Organization for Standardization, Geneva, Switzerland.

[15] Marouani, H. \& Dagenais, M. R. (2008). Internal Clock Drift Estimation in Computer Clusters. Journal of Computer Systems, Networks, and Communications, Volume 2008, Article ID 583162 (2-5). https://doi.org/10.1155/2008/583162

[16] Hewlett Packard, HP 3457A Multimeter Operating Manual, Revised May 1986, 1-11, 3-9.

[17] Hewlett Packard, HP 3457A Multimeter Service Manual (I-4)

[18] American Association for Laboratory Accreditation A2LA, G104 - Guide for Estimation of Measurement Uncertainty in Testing, December 4, 2014 (15)

[19] Fruehauf, H. (2007). Precision Oscillator Overview, Frequency Electronics Inc., 36.

[20] Tavella, P. (2006). Time Scales. MAPAN Journal, 21(4), 217.

Contact information:

Ivica MILANOVIĆ, MSc, Head of Laboratory

Predrag RAKONJAC, MSc, Head of Sector

Technical Test Center

Vojvode Stepe 445A, 11000 Belgrade, Serbia

E-mail: msivica@gmail.com

E-mail: rakonjacp@ptt.rs

Željko BELJIĆ, MSc, PhD student, Research Associate

Zoran MITROVIC, $\mathrm{PhD}$, Professor

University of Novi Sad, Faculty of Technical Sciences

Trg Dositeja Obradovića 6, 21000 Novi Sad, Serbia

E-mail: zbeljic@uns.ac.rs

E-mail: zoranmit@uns.ac.rs 